\title{
Correlation between Anti-Mullerian Hormone Levels and Antral Follicle Numbers in Polycystic Ovary Syndrome
}

\author{
Silva-Vera Marisol ${ }^{1}$, Beltrán-Campos Vicente ${ }^{2}$
}

\begin{abstract}
Introduction: Polycystic Ovary Syndrome (PCOS) is characterized by clinical and biochemical hyperandrogenism, polycystic ovarian morphology, altered gonadotropin secretion, insulin resistance and /or hyperinsulinemia associated with obesity.
\end{abstract}

Objective: The objective of this study was to compare the correlations between levels of FSH, LH, estradiol, Anti-Mullerian Hormone (AMH), and antralfollicle numbers in women with polycystic ovary syndrome.

Methods: PCOS was diagnosed when at least two out of the three parameters were present, according to the Rotterdam criteria. Patients were grouped according to the presence of oligomenorrhea and amenorrhea. Levels of $\mathrm{AMH}, \mathrm{FSH}, \mathrm{LH}$ and Estradiol were determined and a transvaginal ultrasound was performed to determine ovarian volume and the number of antral follicles.

Results: Pearson's correlation revealed a significant correlation $(0.283, p=0.01)$ between $\mathrm{AMH}$ and antralfollicle number. It was appetent that the higher the number of antral follicles the concentrations of $\mathrm{AMH}$ is also higher. A linear correlation showed that the $\mathrm{AMH}$ concentration and the number of antral follicles correlated positively $(r=0.303, p=0.002)$. While the levels of FSH correlated negatively with the number of antral follicles $(r=-0.182, p=0.05)$. The ovarian volume also correlated positively with the number of follicles $(r=0.708, p=0.000)$.

Conclusions: A significant correlation of $\mathrm{AMH}$ with the number of antral follicleswas revealed.

Keywords: polycystic ovary syndrome, anti-Müllerian hormone, ovarian reserve.

\section{INTRODUCTION}

Polycystic ovary syndrome (PCOS) is the most common endocrine disorder in reproductive-aged women with a prevalence of $6 \%-18 \%{ }^{1}$, which is characterized by clinical $^{2}$ and biochemical hyperandrogenism, polycystic ovarian morphology, altered gonadotropin secretion, insulin resistance and/or hyperinsulinemia associated with obesity. ${ }^{3-4}$

\footnotetext{
Department of Nursing and Obstetrics. Division of Health Sciences and Engineering. Celaya-Salvatierra. University of Guanajuato, Mexico.

${ }^{2}$ Department of Clinical Nursing. Division of Health Sciences and Engineering, CelayaSalvatierra. University of Guanajuato, Mexico.
}

Correspondence: Marisol Silva-Vera

E-mail: msol78@gmail.com

Competing interests: None
Several authors have described altered levels of gonadotropins, prolactin and sex steroids in $\mathrm{PCOS}^{5}$. Menstrual disturbances commonly observed in PCOS include oligomenorrhea and amenorrhea. About $70 \%-90 \%$ of women with oligomenorrhea have PCOS while $30 \%-40 \%$ of women with amenorrhea could have $\mathrm{PCOS}^{6}$. More than $63 \%$ of women presenting with symptoms of androgen excess have PCOS and $47 \%$ of them have hormonal alterations. ${ }^{7-8}$

Women with PCOS often seek care for menstrual disturbances, clinical manifestations of hyperandrogenism, and infertility. PCOS is the most common cause of anovulatory infertility. ${ }^{9}$ Women with PCOS are likely to have not only a higher antral follicle count (AFC) but also a greater ovarian volume. ${ }^{10}$

AFC is essential to correlate biochemical and clinical markers to prognosticate infertility and ovarian reserve. Furthermore, women with higher serum levels of Anti-Mullerian Hormone (AMH) have longer menstrual cycles compared with those with lower levels. In females, . $\mathrm{AMH}$ is expressed in granulosa cells of growing follicles up to the antral stage, suggesting an important role in early ovarian folliculogenesis. $\mathrm{AMH}$ is able to inhibit the initiation of primordial follicle growth and may also decrease the sensitivity of antral follicles to follicle-stimulating hormone (FSH). In humans, however, the association between androgens and $\mathrm{AMH}$ remains uncertain, and its exact function in follicular recruitment and long-term effects is not well understood. Serum AMH levels could be used also to identify the girls at risk for PCOS in early adulthood, and allow early prevention by life style counselling. The aim of thisstudy was to compare the correlations between hormone levels of FSH, LH, estradiol, $\mathrm{AMH}$, and antralfollicle numbers of women with polycystic ovary syndrome.

\section{METHODS}

This was a descriptive and analytical study. The selection of patients was randomly done by the Gynecology Service by two specialists. All patients underwent a complete medical history including reproductive background. In the participating Gynecology Service, a medical professional approached potential participants to ascertain interest in the study and obtain informed consent. Assurance was given that consent or refusal to participate would not affect the quality of care or eligibility for any future clinical studies and that all information provided would be confidential to the study team. Ethical approval was provided by the Institutional Ethics Committee of The University of Guanajuato with the 
approval / ID CIDCSIC-1191304.

A total of 50 women were included, between 25-30 years of age, who were diagnosed with PCO according to the Rotterdam criteria. ${ }^{11}$ The PCOS was defined with at least two out of the three criteriawere present. The diagnosis was carried out by two experienced Gynecologists. The patients were separated in to two different groups; patients with oligomenorrhea were placed in group 1 , while group 2 was set up by the patients with amenorrhea. In this study, amenorrhea is described as absence of at least 3 to 6 consecutive menstrual cycles or four or fewer menstrual period per year and oligomenorrhea is described as menstrual cycles fewer than 8 cycles per year, or the duration of the cycle exceeds 35 days.

The determinations of LH, FSH and estrogen levels were taken in the 1-3 daysof the menstrual cycle in patients with oligomenorrhea. The determinations levels of LH, FSH and estrogen were taken any day of the menstrual cycle in patients with amenorrhea.The determinations of levels of $\mathrm{AMH}$ were taken any day of the menstrual cycle in all women, during the second study visit. Patients who had a history of previous ovarian surgery and those who had been taking oral contraceptives were excluded from the present study.

\section{Determination of hormone levels}

Afterat least 12 hours of fasting $10 \mathrm{ml}$ of peripheral venous blood, on any dayof the menstrual cycle, was drawn for determining levels of $\mathrm{AMH}$ by an ultra sensitive ELISA(AMHGenII, Beckman CoulterInc.,Webster,TX, USA) with a sensitivity of $0.08 \mathrm{ng} / \mathrm{mL}$. To determine levels of LH, FSH and estrogen, $10 \mathrm{ml}$ of peripheral venous blood were taken in the 1-3 days of the menstrual cycle of each patient and analyzed by means of radio immunoassay.

\section{Transvaginal ultrasonography (USG)}

Each patient had a USG performed by the same observer, a reproductive biologist with a $5 \mathrm{MHz}$ transvaginal probe by Medison Sono Ace 6000C (Medison, Seoul, Korea). The measurements were performed in real time using the highest possible magnification to observe the ovaries. Transvaginal ultrasound was performed in all participants in the early follicular phase between day 2 and day 3 of the menstrual cycle.

Axes measured were length, thickness and width to calculate ovarian volume $(\mathrm{OV}){ }^{12}$ Ultrasonographic image was considered characteristic of PCOS in the presence of 12 or more follicles 2-9 $\mathrm{mm}$ in diameter located in the periphery of the ovaries by scanning each ovary from the inner to the outer margins in a longitudinal cross section, according to the Rotterdam criteria. The total ovarian volume (OV) was defined as the sum of the right and the left ovarian volumes, and the total antral follicle number was defined as the sum of the right and the left antral follicle count (AFC).

\section{Statistical analysis}

We used the statistical package SPSS version 21.0 for the statistical analysis. Descriptive statistics were used to represent the demographic characteristics of women. We performed Pearson's correlation between the number of antral follicles number and $\mathrm{AMH}$ levels, and a linear correlation between LH, FSH, estradiol, $\mathrm{AMH}, \mathrm{OV}$ and antral follicle number.. In all cases the $p$ value accepted as significant was $<0.05$.

\section{RESULTS}

The demographic and clinical characteristics of oligomenorrhea and amenorrhea patients with PCOS are given in Table 1 . No significant differences in age, body mass index and education. The patients showed differences only in the presence of menstruation as expected.

When the women in the current study were classified according to their menstrual cycle into oligomenorrhea and amenorrhea (Table 2) it was found that FSH andLH concentrations were significantly higher in amenorrhea patients $(p=0.024$ and $p=0.028$, respectively).

Analysis of Pearson's revealed a significant correlation $(0.283, p=0.01)$ between $\mathrm{AMH}$ and the number of antral follicles, which showed that the higher the number of antral follicles more the concentrations of $\mathrm{AMH}$.

AMH correlated positively with the number of antral follicles $(r=0.303$, $\mathrm{p}=0.002)$. FSH negatively correlated

\begin{tabular}{|l|c|c|c|}
\hline \multicolumn{4}{|c|}{ Table 1: Demographic and Clinical Characteristic of the Patients } \\
\hline Variable & $\begin{array}{c}\text { Oligomenorrhea } \\
\mathbf{n}=\mathbf{2 5}\end{array}$ & $\begin{array}{c}\text { Amenorrhea } \\
\mathrm{n}=\mathbf{2 5}\end{array}$ & $\mathbf{p}$ \\
\hline Age(years) & $24.2 \pm 5.2$ & $25.7 \pm 5.0$ & 0.190 \\
\hline BMl(kg/m2) & $26.4 \pm 2.62$ & $19.8 \pm 1.70$ & 0.180 \\
\hline Schooling (years) & $11 \pm 3.5$ & $10 \pm 3.0$ & 0.440 \\
\hline \multicolumn{4}{|l|}{ Data are expressedas mean \pm standard deviation. ${ }^{*} \mathrm{p}<0.05}$. \\
\hline
\end{tabular}

\begin{tabular}{|l|c|c|c|}
\hline \multicolumn{4}{|c|}{ Table2: Endocrine parameters among investigated groups } \\
\hline Hormone & $\begin{array}{c}\text { Oligomenorrhea } \\
\mathrm{n}=\mathbf{2 5}\end{array}$ & $\begin{array}{c}\text { Amenorrhea } \\
\mathrm{n}=\mathbf{2 5}\end{array}$ & $\mathrm{p}$ \\
\hline FSH U/L & $9.1 \pm 4.5$ & $11.8 \pm 4.1$ & 0.024 \\
\hline $\mathrm{LH} \mathrm{U} / \mathrm{L}$ & $17.3 \pm 12.6$ & $29.4 \pm 22.4$ & 0.028 \\
\hline Estradiol ng/ml & $92 \pm 22$ & $85 \pm 11$ & $\mathrm{NS}$ \\
\hline AMH $\mathrm{ng} / \mathrm{ml}$ & $8.8 \pm 2.6$ & $8.5 \pm 2.2$ & $\mathrm{NS}$ \\
\hline Data are expressedas mean \pm standard deviation. ${ }^{*} \mathrm{p}<0.05$. & \\
\hline
\end{tabular}




\begin{tabular}{|l|c|c|c|}
\hline \multicolumn{4}{|c|}{ Table 3: Ultrasonographic volumen parameters } \\
\hline Variable & $\begin{array}{c}\text { Oligomenorrhea } \\
\mathrm{n}=\mathbf{2 5}\end{array}$ & $\begin{array}{c}\text { Amenorrhea } \\
\mathrm{n}=\mathbf{2 5}\end{array}$ & $\mathbf{p}$ \\
\hline Number of antralfollicles & $32(25-46)$ & $36(28-57)$ & 0.001 \\
\hline OvarianVolume & $5.0(2.3-9.2)$ & $6.7(3.9-12.4)$ & 0.001 \\
\hline \multicolumn{2}{|l}{} \\
\hline
\end{tabular}

with the number of antral follicles in both groups $(r=-0.182, p=0.05)$.

The serum AMH level was not significantly related to the ovarian volume on ultrasonography.

Ovarian volume positively correlated with the number of follicles $(r=0.708$, $\mathrm{p}=0.000$ ) (Table 3).

\section{DISCUSSION}

In this study, we evaluated the relationship between levelsof $\mathrm{FSH}$, $\mathrm{LH}$, estradiol, $\mathrm{AMH}$, and antral follicle numbers and ovarian volume in women with polycystic ovary syndrome. The results showed a significant correlation between serum $\mathrm{AMH}$ level and the number of antral follicles, which showed that the higher the number of antral follicles more the concentrations of $\mathrm{AMH}$.

Some authors have reported that the highly significant relationship between the serum AMH level and AFC. ${ }^{13-14} \mathrm{AMH}$ is regarded as the most useful marker of the status of ovarian reserve. ${ }^{15}$

Swellam et al. Other authors suggest that, serum AMH levels might be introduced as a marker to be utilized clinically in the differential diagnosis of hyperandrogenemic patient ${ }^{16}$. Our data confirm these findings, which showed that higher the number of antral follicles the more the concentration of $\mathrm{AMH}{ }^{16}$

Patients who were diagnosed with PCOS- Amenorrhea showed elevated levels of $\mathrm{FSH}$ and $\mathrm{AMH}$, ovarian volume and number of antral follicles. In addition it has been reported that AMH levels between hyperandrogenic and non-hyperandrogenic PCOS patients are different. ${ }^{17}$ In the current study elevated FSH levels were reported in oligomenorrhea patient with PCOS.
Increased LH and normal to low FSH such as young PCOS women with hyperandrogenemia, serum $\mathrm{AMH}$ levels are increased, ${ }^{18}$ this was seen in our study.

In contrast, we found not significant correlation between the serum AMH level and the ovarian volume, and this result is in disagreement with that of a previous study showing that the $\mathrm{AMH}$ levels positively correlate with the ovarian volume. ${ }^{19}$ One possible explanation is that the ovarian stromal volume is not significantly affected by the serum AMH level.

Hormone levels of FSH showed significant differences in the two groups. Stimulatory effect of FSH on AMH secretion in oligo/anovulatory PCOS women is known. CatteauJonard and Dewailly, suggest that the defective selection of a dominant follicle in anovulatory patients, results in a local inhibition of FSH action. ${ }^{20}$

Current tests to determine the ovarian response, which include $\mathrm{AMH}$ are designed to predict how a woman can respond to controlled ovarian stimulation, so it is considered that assessing functional ovarian reserve through $\mathrm{AMH}$ is not enough.FSH levels increase with age, hindering the estimation of ovarian reserve and decrease possible fertility. The number of antral follicles, an indirect measure of ovarian reserve, seen through a transvaginal ultrasound is now reliable and allows us to have a more accurate estimation even when it comes to starting a treatment protocol for infertility. Antral follicle count with transvaginal sonography is a noninvasive and easy to perform method that provides predictive information about ovarian responsiveness before stimulation. $^{21}$ Further research is needed to determine the relationship of $\mathrm{AMH}$ and PCOS. AMH may be a useful initial diagnostic test for PCOS subject to validation in prospective population..$^{22}$ The study was restricted to women who sought treatment for infertility. Moreover, we believe that AMH can be used as a marker in conjunction with ultrasound values to assess ovarian reserve in the future.

\section{ACKNOWLEDGEMENTS}

This work was supported by the University of Guanajuato.

\section{AUTHOR CONTRIBUTIONS}

Silva-Vera M conceived and designed the study, performed the experiments and data analysis, and drafted the manuscript. Beltran-Campos V carried out the hormonal analysis. All authors have read and approved the final manuscript.

\section{REFERENCES}

1. Moran C, Tena G, Moran S, Ruiz P, Reyna R, Duque X. Prevalence of polycystic ovary syndrome and related disorders in women. GynecolObstet Invest 2010; 69:274-280.

2. Swellam M, Khaial A, Mosa T, El-Baz H, Said M. Anti-mullerian and androgens hormones in women with polycystic ovary syndrome undergoing IVF/ICSI. Iran J Reprod Med.2013;11(11):883-90.

3. Usadi RS, Legro RS. Reproductive impact of polycystic ovary syndrome. CurrOpinEndocrinol Diabetes Obes. 2012 Dec;19(6):505-11. doi: 10.1097/ MED.0b013e328359ff92.

4. Burt Solorzano CM, Beller JP, Abshire MY, Collins JS, McCartney CR, Marshall JC. Neuroendocrine dysfunction in polycystic ovary syndrome. Steroids. 2012 Mar 10;77(4):332-7. doi: 10.1016/j. steroids.2011.12.007.

5. Dewailly D, Gronier H, Poncelet E, Robin G, Leroy M, Pigny P, Duhamel $A$, Catteau-Jonard $S$. Diagnosis of polycystic ovary syndrome (PCOS): revisiting the threshold values of follicle count on ultrasound and of the serum AMH level for the definition of polycystic ovaries.HumReprod. 2011;26(11):31239. doi: 10.1093/humrep/der297.

6. Fauser B, Tarlatzis B, Rebar R, et al. 
Consensus on women's health aspects of polycystic ovary syndrome (PCOS): the Amsterdam ESHRE/ASRM-Sponsored 3rd PCOS Consensus Workshop Group. FertilSteril. 2012;97(1):28-38.

7. Vargas CM, Gabriel SB, Herrera-PJ. Síndrome de ovarios poliquísticos: abordaje diagnóstico y terapéutico. RevBiomed 2003;14:191-203.

8. Ibáñez Hernández L, Mora Pérez J, Paredes Palma JC. Prevalencia de síndrome de ovario poliquístico en pacientes con cáncer de mama y diabetes ¿posible factor de riesgo? Revista de Especialidades Médico-Quirúrgicas 201217266-272.

9. Parco S, Novelli C, Vascoto F. Serum antiMullerian hormone as a predictivemarker of polycysticovariansyndrome.Int J Gen Med.2011;4:759-763.

10. Chun S. Serum luteinizing hormone level and luteinizing hormone/ follicle-stimulating hormone ratio but not serum anti-Müllerian hormone level is related to ovarian volume in Korean women withpolycystic ovary syndrome. ClinExpReprod Med.2014; 41 (2): 86-91.http://dx.doi.org/10.5653/ cerm.2014.41.2.86

11. The Rotterdam ESHRE/ASRMSponsored PCOS consensus workshop group. Revised 2003 consensus on diagnostic criteria and long-term health risks related to polycystic ovary syndrome (PCOS). Hum Reprod2004;19:41-47. doi:10.1093/humrep/deh098.
12. Porter MB. Polycystic ovary syndrome: the controversy of diagnosis by ultrasound. SeminReprod Med. 2008;26(3):241-51. doi: 10.1055/s-2008-1076143.

13. Iliodromiti S, Kelsey TW, Anderson RA, Nelson SM. Can anti-Mullerian hormone predict the diagnosis of polycystic ovary syndrome? A systematic review and meta-analysis of extracted data. J ClinEndocrinolMetab 2013;98:33323340.

14. Homburg R, Ray A, Bhide P, Gudi A, Shah A, Timms $P$, et al. The relationship of serum anti-Mullerian hormone with polycystic ovarian morphology and polycystic ovary syndrome: a prospective cohort study. Hum Reprod 2013;28:10771083.

15. Felicia Yarde. Et.al. Anti-müllerian hormone as predictor of reproductive outcome in subfertile women with elevated basal follicle-stimulating hormone levels: a follow-up study. Fertility and Sterility. 2013. dx.doi.org/10.1016/j. fertnstert.2013.05.009

16. West $S$, Lashen $H, B l o i g u ~ A$, Franks $S$, Puukka K, Ruokonen $A$, Järvelin MR, Tapanainen JS, Morin-Papunen L. Irregular menstruation and hyperandrogenaemia in adolescence are associated withpolycystic ovary syndromeand infertility in later life: Northern Finland Birth Cohort 1986 study. Hum Reprod. 2014, pii: deu200.

17. 17. Sahmay $S$, Aydın $Y$, Atakul $N$, Aydogan B, Kaleli S. Relation of antimullerian hormone with the clinical signs of hyper and rogenism and polycystic ovary morphology. Gynecol Endocrinol. 2014;30(2): 130-4. doi: 10.3109/09513590. 2013.867320.

18. Koutlaki N, Dimitraki $M$, Zervoudis S, Poiana C, Psillaki A, Nikas I, Liberis A, Badiu C, Liberis V. The relationship between Anti-Müllerian hormone and other reproductive parameters in normal women and in women with polycystic ovary syndrome. J Med Life. 2013;6(2):146-50.

19. You SY, Park SY, Yang GY, Jeong KA, Kim YJ, Chung HW. Anti-Mullerian hormone in women with polycystic ovary syndrome. Korean J ObstetGynecol 2012;55:315-324.

20. Catteau-Jonard S, Dewailly D. Pathophysiology ofpolycystic ovary syndrome: the role of hyperandrogenism. Front Horm Res.2013;40:22-7. doi: $10.1159 / 000341679$.

21. Adibi A, Mardanian F, Hajiahmadi S. Comparison of Ovarian volume and Antral follicle count with Endocrine tests for prediction of responsiveness in ovulation induction protocols. Adv Biomed Res.2012;1:71. doi: 10.4103/22779175.102975.

22. Iliodromiti S, Kelsey TW, Anderson RA, Nelson SM. Can anti-Mullerian hormone predict the diagnosis of polycystic ovary syndrome? A systematic review and meta-analysis of extracted data. $J$ ClinEndocrinolMetab.2013;98(8): 333240. doi: 10.1210/jc.2013-1393. 